\title{
COMPARISON OF THE EFFECT OF DIFFERENT IMPLANT CONNECTIONS ON STRESS DISTRIBUTION AROUND DENTAL IMPLANTS (IN VITRO STUDY)
}

\author{
Moustafa Mahmoud Soliman* , Nevine Hassan Kheir El Din ${ }^{* *}$ and Mohamed Wagdy Bissar ${ }^{* * *}$
}

\begin{abstract}
Statement of the problem: In implant dentistry literature, the most commonly investigated materials/structures in finite element analysis studies are either implant, peri-implant bone (cortical and cancellous bone), and restoration. This method allows application of simulated forces at specific points in the system and analysis of stress in the implant and peri-implant region. The implant connection design and other factors as the prosthesis type, its height and material have a great effect on stresses falling on the bone around implant
\end{abstract}

Objectives: to evaluate and compare stress distribution using (3dimensional) finite element analysis between implants with Internal Hex and Morse Taper connections that were simulated in bone without vertical defect and with $6 \mathrm{~mm}$ vertical defect.

Material and methods: In this study, the implants examined were titanium implants with Morse taper and internal hex connections. These implants were utilized in the two configurations that were designed in this study. In the first configuration, the jawbone has no vertical defect and composed of (starting from top down) one mm layer of crestal cortical bone, $3.5 \mathrm{~mm}$ layer of cancellous bone, $0.5 \mathrm{~mm}$ sinus cortical bone. In the second configuration, it is composed of (starting from top down) one $\mathrm{mm}$ layer of crestal cortical bone, $3.5 \mathrm{~mm}$ layer of cancellous bone, $0.5 \mathrm{~mm}$ sinus cortical bone with a $6 \mathrm{~mm}$ vertical bone defect was created. The force of $100 \mathrm{~N}$ is applied in axial, oblique and axial and oblique directions. Stresses falling on surrounding bone, fixture, abutment and screw were analyzed using finite element analysis in the two different configurations.

Results: The maximum Von Mises stresses for different loading conditions were recorded at different areas of the implant abutment assembly and in the surrounding bone and it was found that in all direction of forces, the stress levels falling on the bone, abutment, fixture and screw were more in the bone defect configuration than the models with no bone defects.In all direction of forces, the implants with Morse taper abutments showed less stress on the fixture compared to internal hex abutments in both models with and without defect.

Conclusion: Morse taper connections showed less stresses on the bone, fixture, abutment and screw than the internal Hex connection regardless of the bone height. Furthermore, stress distribution was more with implant in the $6 \mathrm{~mm}$ vertical defect than that in the bone without defect.

\footnotetext{
* BDS Faculty of Dentistry Alexandria University, MSc Periodontology Department Ain Shams University.

** Professor of Oral Medicine, Periodontology and Oral Diagnosis, Faculty of Dentistry, Ain Shams University

*** Lecturer of Oral Medicine, Periodontology and Oral Diagnosis, Faculty of Dentistry, Ain Shams University
} 


\section{INTRODUCTION}

Having sufficient volume of healthy bone for long-term success of osseointegrated implants is not always present as a result of trauma, tooth loss or infectious diseases such as advanced periodontitis which leads to vertical bone defects. ${ }^{(1)}$

The implant-abutment connections more commonly used today are screw-retained and can be divided into two major groups: external and internal connections. The most widely used external connection is the 'external hexagon', originally used on the Branemark implant.-However, due to mechanical problems including screw loosening and the concern for better aesthetics led to the development of internal connections-(2)

Several methods based on photoelastic, straingauge, and finite element analysis (FEA)-based studies have been used to investigate stress in the peri-implant region and in the components of implant-supported restorations. ${ }^{(3-6)}$

FEA is a numerical stress analysis technique that is widely used to assess engineering and biomechanical problems before they occur. A finite element model is constructed by dividing solid objects into several elements that are connected at a common nodal point. Each element is assigned appropriate material properties corresponding to the properties of the object being modeled. The first step is to subdivide the complex object geometry into a suitable set of smaller 'elements' of 'finite' dimensions. When combined with the 'mesh' model of the investigated structures, each element can adopt a specific geometric shape (i.e., triangle, square, tetrahedron, etc.) with a specific internal strain function. Using these functions and the actual geometry of the element, the equilibrium equations between the external forces acting on the element and the displacement occurring at each node can be determined. ${ }^{(7,8)}$

In implant dentistry literature, the most common investigated materials/structures in FEA studies can be classified as either implant, peri-implant bone (cortical and cancellous bone), and restoration.
This method allows application of simulated forces at specific points in the system and stress analysis in the peri-implant region and surrounding structures. ${ }^{(9-12)}$

The implant-abutment connection is believed to play an important role in the outcome of the implant therapy. Bacterial leakage through the implantabutment interface microgap and colonization of the connection's inner portion determine the formation of the peri-implant chronic inflammatory infiltrate, thereby leading to bone resorption, ${ }^{(13)}$

Additionally, the literature shows that the stress/ strain concentration induced by an excessive, dynamic loading can trigger bone resorption, by bone microdamage accumulation around osseointegrated implants, even in the absence of an oral biofilm . ${ }^{(14)}$

Regarding the survival rate of the of implants after ridge augmentation, the analysis shows that the implant survival is comparable whereas the success rate is not comparable with dental implants which are placed in non-augmented bone..$^{(15)}$

Thus, the present study was conducted to calculate the distribution of stresses on both implant components and the surrounding bone with finite element analysis in internal hex versus Morse taper connections placed at different bone heights.

\section{MATERIAL AND METHODS}

The implant examined throughout this study is a virtual titanium implant of $10 \mathrm{~mm}$ length and 4.5 $\mathrm{mm}$ width in both configurations and the abutments are internal hex and Morse taper of $7 \mathrm{~mm}$ length in the first configuration and $12 \mathrm{~mm}$ in the second configuration. In order to model the surrounding bone, two different configurations were used. In the first configuration, the jawbone has no defects. It is composed, (starting from top to bottom down) of a layer $(1 \mathrm{~mm})$ of crestal cortical bone, a layer of $3.5 \mathrm{~mm}$ cancellous bone, a sinus $0.5 \mathrm{~mm}$ cortical bone. (Figure 1) In the second configuration, a vertical bone defect of $6 \mathrm{mms}$ was created. (Figure 2).

All the components were meshed with solid 92 


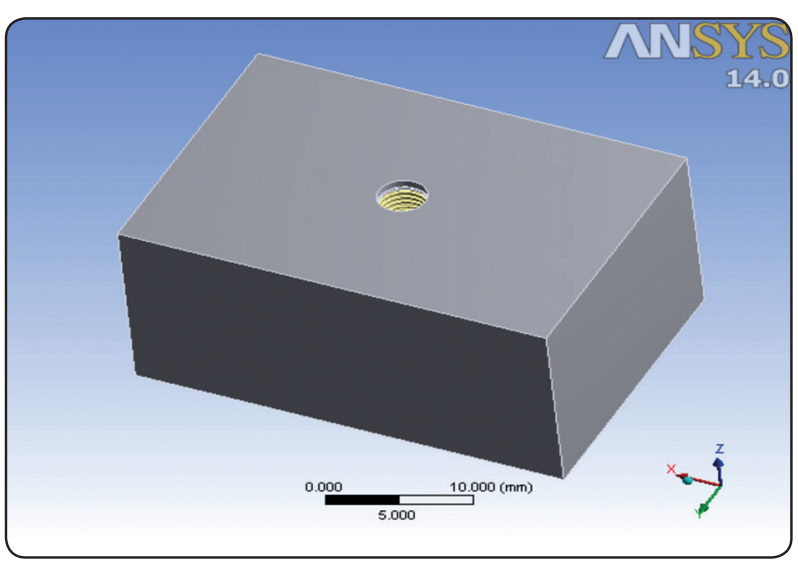

Fig. (1) $1^{\text {st }}$ configuration, no bone defect

elements. Solid 92 is a $2^{\text {nd }}$ order tetra element which has 10 nodes (Table 1). Solid 92 has a quadratic displacement behavior and are well suited to model irregular surfaces. This meshed model was then imported into ANSYS 13 software (Ansys Inc. USA) to perform the numerical simulation.

Table (1) Nodes and elements of each component

\begin{tabular}{|l|l|l|l|l|}
\hline & & Hex & Morse & Defect \\
\hline \multirow{3}{*}{ Abutment } & Nodes & 40886 & 39656 & 51463 \\
\cline { 2 - 5 } & Elements & 27066 & 26027 & 34379 \\
\hline \multirow{3}{*}{ Fixture } & Nodes & 45624 & 42708 & 42815 \\
\cline { 2 - 5 } & Elements & 28989 & 26742 & 26805 \\
\hline \multirow{3}{*}{ Screw } & Nodes & 31104 & 30564 & 31104 \\
\cline { 2 - 5 } & Elements & 20205 & 19804 & 20205 \\
\hline \multirow{3}{*}{ Spongy } & Nodes & 121241 & 122745 & 83033 \\
\cline { 2 - 5 } & Elements & 72709 & 73804 & 46918 \\
\hline \multirow{3}{*}{ Total } & Nodes & 195741 & 194148 & 222078 \\
\cline { 2 - 5 } & Elements & 135762 & 134634 & 155065 \\
\cline { 2 - 5 } & Nodes & 510841 & 513752 & 525733 \\
\hline
\end{tabular}

The properties of the different materials used throughout this study are listed in Table 2 .

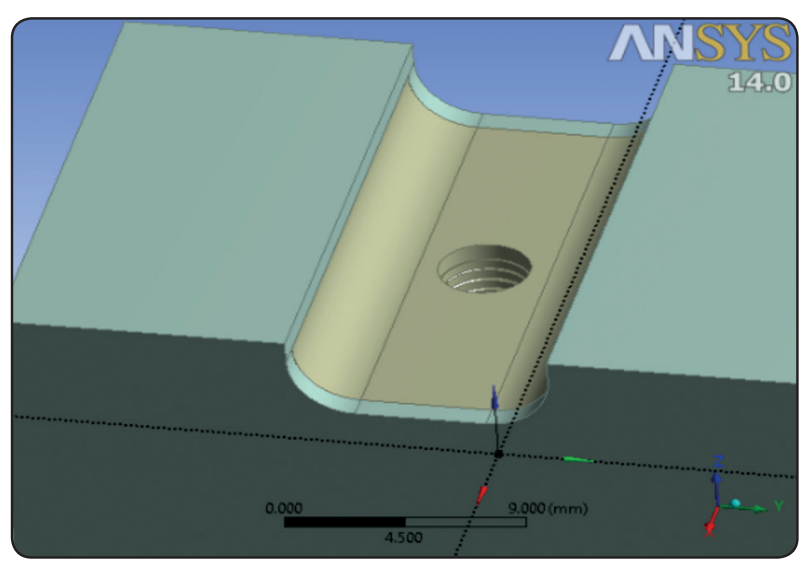

Fig. (2) $2^{\text {nd }}$ configuration, vertical bone defect

Table (2) The properties of the different materials used throughout this study

\begin{tabular}{|c|c|c|}
\hline Material & Elastic modulus (GPa) & Poisson's ratio \\
\hline $\begin{array}{c}\text { Trabecular } \\
\text { bone }\end{array}$ & 1.37 & 0.30 \\
\hline Cortical bone & 13.7 & 0.30 \\
\hline Titanium & 110 & 0.35 \\
\hline
\end{tabular}

Depending on the hardness of foods, the average bite force reportedly ranges from 20 to $120 \mathrm{~N}$. In the present study, we applied a static load of $100 \mathrm{~N}$ to simulate loading by occlusion as indicated in previous studies..$^{(16-18)}$ forces are applied in 3 directions to simulate the actual forces in mouth which are a- Axial, b- Oblique, c- Axial and oblique.

Stresses on each model are evaluated according to the stress values from low to high. Frequently, different colors represent the amount of stress around periimplant regions and prosthetic structures.

\section{RESULTS}

The data obtained from the ANSYS calculation is presented in a stress distribution map with a color scale (Figure 3) which allowed us to compare the stress levels in the various components of the models. In this study, the maximum Von Mises stresses for different loading conditions were recorded at different areas of the implant abutment assembly and in the surrounding bone. 


\section{Effect on Bone}

In all force directions, the stress level was more in the bone defect configuration than the models with no bone defects. Moreover, the implants with Morse taper abutments showed less stress on the bone compared to internal hex abutments in all directions of force and both models with and without defect (Figure 4).

\section{Effect on Abutments}

In all direction of forces, the stress level on the abutment was more in the bone defect configuration than the models with no bone defects.

In all directions of forces, the implants with Morse taper abutments showed less stress on the abutment compared to internal hex abutments in both models with and without defect (Figure 5).

Regarding the stress concentration areas it was found that the highest stresses were concentrated at the submarginal (the collar part) of the abutment in both models in all directions of forces except for the hex connection in the defect model it was concentrated at the middle third of the abutment (supramarginal).

\section{Effect on Fixture}

For all direction of forces, the stress level on the fixture was more in the bone defect configuration than the models with no bone defects.

In all direction of forces, the implants with Morse taper abutments showed less stress on the fixture compared to internal hex abutments in both models with and without defect (Figure 6).

The stress was concentrated at the inner surface of the fixture where it is in contact with the external surface of the abutment in both models in all directions except for the axial load direction in defect model for both Morse and hex abutment it was concentrated at the external surface of the last thread of the fixture.

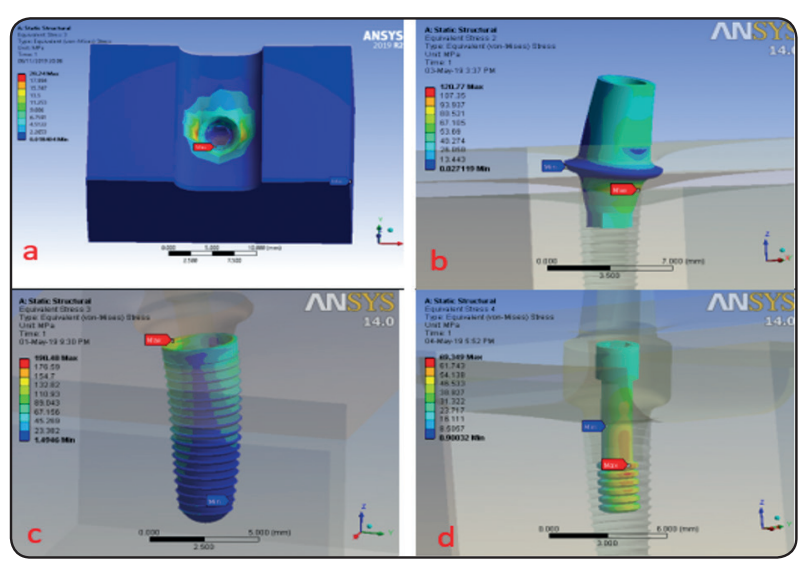

Fig. (3) FEA of the model showing example of stresses on (a) bone, (b) abutment, (c) fixture and (d) screw

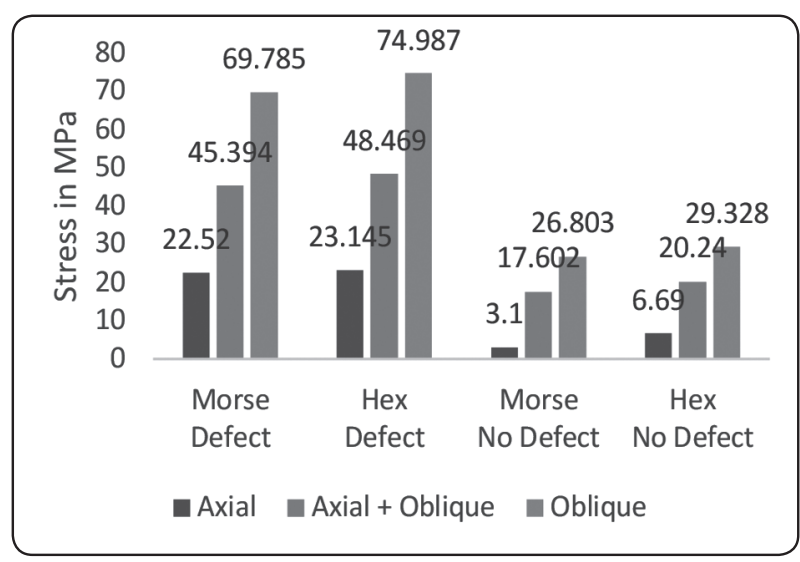

Fig. (4) Stress level on the bone

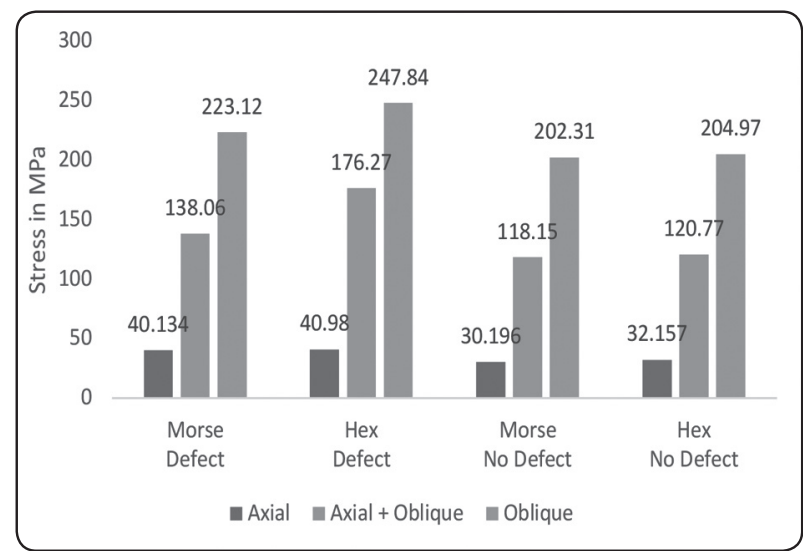

Fig. (5) Stress levels on the abutments 


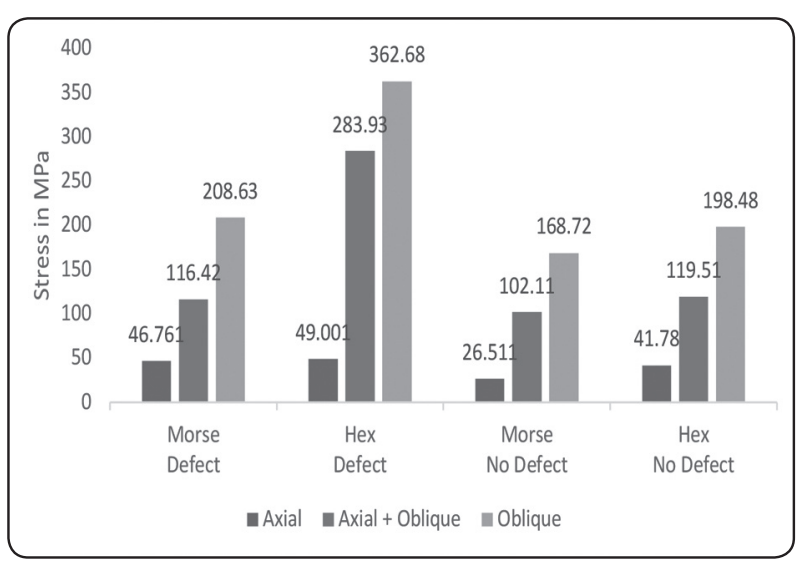

Fig. (6) Stress levels on fixture

\section{Effect on the Screw}

In all directions of forces, the stress level on the screw was more in the bone defect configuration than the models with no bone defects.

In all directions of forces, the implants with Morse taper abutments showed less stress on the screw compared to internal hex abutments in both models with and without defect (Figure 7). It was also found that the maximum stresses were recorded between the shank and threads of the screw in all models and all directions of forces.

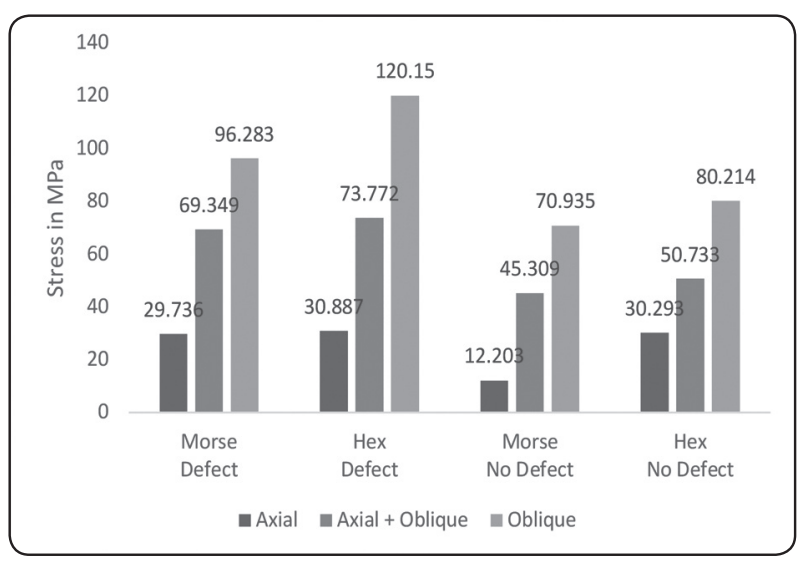

Fig. (7) Stress levels on the screw

\section{DISCUSSION}

A key factor for the success of a dental implant is the way stresses are transferred to the surrounding bone which depends on the implant connection system and other factors as the type of loading, the bone-implant interface, the length and diameter of the implants, the shape and characteristics of the implant surface and the quantity and quality of the surrounding bone..$^{(19)}$

Vertical defects augmentation have a very high risk for complications, like soft tissue dehiscence, infection, less bone quantity, and failure of the graft. Although many techniques have been suggested to deal with the functional and esthetic problems, there is still high risk of failure. ${ }^{(20)}$ Therefore this study aimed to find an alternative for augmentation by using long abutments directly in the level of crestal bone avoiding the vertical cantilever stresses.

The FEA has been chosen for this study as it has been an effective method for investigating the behavioral tendencies and predicting stress distribution on implants and surrounding bone under loading conditions. ${ }^{(21)}$

Regarding the results of this study, the stress level was more in the bone defect configuration than the models with no bone defects. This could be attributed to the length of the fulcrum arm which is longer in case of vertical defect. Moreover, the implants with Morse taper abutments showed less stress on the bone compared to internal hex abutments in all directions of force and in both models with and without defects. This could be attributed to the implant abutment interface (IAI) in the conical connections that allows better stress distribution than the hex connections.

Saidin et al ${ }^{(22)}$ found that the peak stress in the bone supporting a dental implant in FEA appears in the crestal region close to the level where the implant attaches with bone. The smaller the peak stress caused by a standardized load, the greater the load required to trigger bone fracture or bone resorption. It has also been stated in literature that 
the IAI affects the stresses in the marginal bone only when it is located close to the marginal bone. This was in agreement with the current study results where the stresses were concentrated at the marginal area.

Another study revealed that the elements exposed to maximum stress were located where most of the non-axial masticatory forces were transferred. This situation corresponds to nonparametric computerized models of loaded dental implants. Models show utmost strain acting around the implant neck. This is the location where bone overloading occurs. ${ }^{(23)}$ This was in agreement with the current study results.

It is widely debated that implant-abutment connection designs can induce different degrees of crestal bone remodeling after being subjected to functional loading. Pozzi et al (24) showed that marginal bone loss was statistically significantly different, with better results for the internal conical connection, compared to external hex. This also supports the current study findings.

Similar to the bone the results the stress level on the abutment was more in the bone defect configuration than the models with no bone defects. In all directions of forces, the implants with Morse taper abutments showed less stress on the abutment compared to internal hex abutments in both models with and without defect.

In agreement with the obtained results, an ideal implant abutment design should avoid micro-gaps and simultaneously keep the marginal bone strain as low as possible. Saidin et al ${ }^{(22)}$ indicated in that the maximum permissible physical value of bone strain is $3000 \mu \mathrm{m} / \mathrm{mm}$ and the internal hexagon connections produced greater stress on the bone and the lower stresses on the connection assembly.

Hansson et al ${ }^{(25)}$ concluded that the IAI has a profound effect on the stress state in the marginal bone and that the conical interface can resist a larger axial load than the implant with flat top interface. This supports the current study findings.
The results of the present study revealed that the stress level on the fixture was more in the bone defect configuration than the models with no bone defects. In all directions of forces, the implants with Morse taper abutments showed less stress on the fixture compared to internal hex abutments in both models with and without defect. This could be attributed to the inner slopes of the fixture contacting the outer slopes of the abutment in Morse taper resulting in less stresses.

In agreement with the obtained results, Coppedê et al (26) found that maximum deformation force for conical implants was statistically higher than that for internal hex. Fracture force for the internal hex implants was $79.86 \pm 4.77 \mathrm{kgf}$ while none of the conical implants fractured. The friction-locking mechanics and the solid design of the conical abutments provided greater resistance to deformation and fracture under oblique compressive loading when compared to the internal hex abutments.

On the other hand, Sarafaraz et al (27) found that the two conical connections had a friction type of contact between the slopes of the outer surface of the abutment and inner surface of the fixture. Whereas the tri-channel connection (TCC) and parallel hex connection (PHC) showed only a close approximation of the outer parallel surface of the abutment and the parallel internal walls of the implant and the abutment rested on the outer periphery of the head of the implant. This resulted in the maximum amount of Von Mises stresses recorded for the conical connections on the inner surface of the implant and less than half of these stresses recorded for the TCC and PHC at the external periphery on the head of the implant. The difference in results could be attributed to the difference in the design of the model used in each of the two studies.

Regarding the effect on the screw, the current study showed more stresses in bone defect models than the non-defective models, and that could be due to the higher stresses falling on the abutment in 
the defect models due to the length of the fulcrum arm which is longer in case of vertical defect and transferred to the screw. In all directions of forces, the implants with Morse taper abutments showed less stress on the screw compared to internal hex abutments in both models with and without defect. This could be attributed to the better stress distribution for the Morse taper connection compared to the Hex connection.

This was further confirmed from a study which proved that dental implants with the Morse taper connection have been designed to establish a better stress distribution when lateral external forces act on the prosthesis and minimize the forces transmitted to the fastening screw. ${ }^{(19)}$

In a 3D FEM analysis the maximum stress was recorded between the shank and first thread of all implant systems, and stress increased under loading conditions which was in agreement with the obtained results. ${ }^{(28)}$

From a systematic review, it was concluded that if a proper anti-rotational feature and torque are employed, then the abutment screw loosening is a rare occurrence regardless of the IAI geometry. (29) This demonstrated that Morse taper abutment is preferable than Hex connection as it does not rely only on the screw.

On the controversy, Segundo et $\mathrm{al}^{(30)}$ stated that the internal connection protects the abutment screw from stresses and the screw material did not show any influence on stress concentration. They also did not identify the most harmful loading conditions.

There are limitations in this study some of which are universal to all FEAs such as the interface between the implant and the cortical or cancellous bone were completely bonded which may not be the case in clinical conditions and all the implant abutment connections were assumed to be bonded with each other which may not be the exact simulation of the clinical condition.

\section{CONCLUSIONS}

Within the limitations of the present study, we conclude that the stress distribution was more in implant in $6 \mathrm{~mm}$ vertical defect than without defect. Moreover, Morse taper connections showed less stresses on the bone, abutment, fixture and screw than the internal Hex connection regardless of the bone height.

\section{Statement of Conflict of Interest}

The authors declare that they have no conflicts of interest.

\section{REFERENCES}

1. Mecall RA, Rosenfeld AL. Influence of residual ridge resorption patterns on implant fixture placement and tooth position. 1. Int $\mathrm{J}$ Periodontics Restorative Dent. 1991;11(1):8-23.

2. Gracis S, Michalakis K, Vigolo P, Vult von Steyern P, Zwahlen M, Sailer I. Internal vs. external connections for abutments/reconstructions: a systematic review. Clin Oral Implants Res. 2012;23 Suppl 6:202-16.

3. Assuncao WG, Barao VA, Tabata LF, Gomes EA, Delben JA, dos Santos PH. Biomechanics studies in dentistry: bioengineering applied in oral implantology. The Journal of craniofacial surgery. 2009;20(4):1173-7.

4. Geng JP, Tan KB, Liu GR. Application of finite element analysis in implant dentistry: a review of the literature. $\mathrm{J}$ Prosthet Dent. 2001;85(6):585-98.

5. Shen WL, Chen CS, Hsu ML. Influence of implant collar design on stress and strain distribution in the crestal compact bone: a three-dimensional finite element analysis . Int J Oral Maxillofac Implants. 2010;25(5):901-10.

6. Srinivasan M, Padmanabhan TV. Intrusion in implanttooth-supported fixed prosthesis: an in vitro photoelastic stress analysis. Indian journal of dental research : official publication of Indian Society for Dental Research. 2008;19(1):6-11.

7. Rieger MR, Fareed K, Adams WK, Tanquist RA. Bone stress distribution for three endosseous implants. J Prosthet Dent. 1989;61(2):223-8.

8. DeTolla DH, Andreana S, Patra A, Buhite R, Comella B. Role of the finite element model in dental implants. J Oral Implantol. 2000;26(2):77-81. 
9. Georgiopoulos B, Kalioras K, Provatidis C, Manda M, Koidis P. The effects of implant length and diameter prior to and after osseointegration: a 2-D finite element analysis. J Oral Implantol. 2007;33(5):243-56.

10. Juodzbalys G, Kubilius R, Eidukynas V, Raustia AM. Stress distribution in bone: single-unit implant prostheses veneered with porcelain or a new composite material. Implant Dent. 2005;14(2):166-75.

11. Meric G, Erkmen E, Kurt A, Eser A, Ozden AU. Biomechanical comparison of two different collar structured implants supporting 3-unit fixed partial denture: a 3-D FEM study. Acta Odontol Scand. 2012;70(1):61-71.

12. Erkmen E, Meric G, Kurt A, Tunc Y, Eser A. Biomechanical comparison of implant retained fixed partial dentures with fiber reinforced composite versus conventional metal frameworks: a 3D FEA study. J Mech Behav Biomed Mater. 2011;4(1):107-16.

13. King, G. N., Hermann, J. S., Schoolfield, J. D., Buser, D., \& Cochran, D. L. (2002). Influence of the size of the microgap on crestal bone levels in non-submerged dental implants: a radiographic study in the canine mandible. $\mathrm{J}$ Periodontol, 1111-1117. doi: 10.1902/jop.2002.73.10.1111

14. Duyck, J., Ronold, H. J., Van Oosterwyck, H., Naert, I., Vander Sloten, J., \& Ellingsen, J. E. (2001). The influence of static and dynamic loading on marginal bone reactions around osseointegrated implants: an animal experimental study. Clin Oral Implants Res, 207-218.

15. Motamedian, S. R., Khojaste, M., \& Khojasteh, A. (2016). Success rate of implants placed in autogenous bone blocks versus allogenic bone blocks: A systematic literature review. Annals of maxillofacial surgery, 78-90. doi: 10.4103/2231-0746.186143

16. Eskitascioglu G, Usumez A, Sevimay M, Soykan E, Unsal E. The influence of occlusal loading location on stresses transferred to implant-supported prostheses and supporting bone: A three-dimensional finite element study. J Prosthet Dent. 2004;91(2):144-50.

17. Sagat G, Yalcin S, Gultekin BA, Mijiritsky E. Influence of arch shape and implant position on stress distribution around implants supporting fixed full-arch prosthesis in edentulous maxilla. Implant Dent. 2010;19(6):498-508.

18. Bayraktar M. The influence of crown-implant ratio and dental implant parameters on implant and periimplant bone: A finite element analysis. . Institute of Health Science: Istanbul University; 2011.

19. Borie E, Orsi IA, Yoshito Noritomi P, Takanori Kemmoku D. Three-Dimensional Finite Element Analysis of the Biomechanical Behaviors of Implants with Different Connections, Lengths, and Diameters Placed in the Maxillary Anterior Region. Int J Oral Maxillofac Implants. 2016;31(1).
20. Öncü E, Isik K, Alaaddinoğlu EE, Uckan S. Combined use of alveolar distraction osteogenesis and segmental osteotomy in anterior vertical ridge augmentation. International journal of surgery case reports. 2015;8:124-6.

21. Omori M, Sato Y, Kitagawa N, Shimura Y, Ito M. A biomechanical investigation of mandibular molar implants: reproducibility and validity of a finite element analysis model. International journal of implant dentistry. 2015;1(1):10

22. Saidin S, Kadir MRA, Sulaiman E, Kasim NHA. Effects of different implant-abutment connections on micromotion and stress distribution: Prediction of microgap formation. Journal of Dentistry. 2012;40(6):467-74.

23. Xing Z, Chen LS, Peng W, Chen LJ. Influence of Orbital Implant Length and Diameter on Stress Distribution: A Finite Element Analysis. The Journal of craniofacial surgery. 2017;28(2):e117.

24. Pozzi A, Agliardi E, Tallarico M, Barlattani A. Clinical and radiological outcomes of two implants with different prosthetic interfaces and neck configurations: randomized, controlled, split-mouth clinical trial. Clinical implant dentistry and related research. 2014 Feb;16(1):96-106.

25. Hansson S. Implant-abutment interface: biomechanical study of flat top versus conical. Clin Implant Dent Relat Res. 2000;2(1):33-41.

26. Coppedê AR, Bersani E, de Mattos MD, Rodrigues RC, de Mattias Sartori IA, Ribeiro RF. Fracture resistance of the implant-abutment connection in implants with internal hex and internal conical connections under oblique compressive loading: an in vitro study. International Journal of Prosthodontics. 2009 May 1;22(3).

27. Sarfaraz, Hasan \& Shenoy, Kamalakanth \& Hussain, Akhter \& Sucheta, P. (2015). A Comparison of Four Implant Abutment Connection Designs on the Stress Distribution Pattern us7ing 3D Finite Element Analysis. International Journal of Oral Implantology \& Clinical Research. 6. 1-8. 10.5005/jp-journals-10012-1128.

28. Deepa R, Surendra Kumar G, Satish Babu C, Shetty S, Jnandev K, Rohit P. Influence of occlusal forces on stress distribution on preloaded dental implant abutment screw: A finite element analysis. Int J Oral Implant Clin Res. 2013;4:16-23.

29. Theoharidou A, Petridis HP, Tzannas K, Garefis P. Abutment screw loosening in single-implant restorations: a systematic review. International Journal Oral \& Maxillofacial Implants. 2008;23(4):681-90.

30. Segundo R, Oshima H, da Silva I, Burnett Jr L, Mota EG, Silva LL. Stress distribution of an internal connection implant prostheses set: A 3D finite element analysis. Stomatologija. 2009;11(2):55-9. 